\title{
Coexistence of Chronic Constrictive Pericarditis can make the Echocardiographic Diagnosis of Atrial Septal Defect Challenging
}

\author{
Sambhunath Das, Randhir Singh Rajput
}

\begin{abstract}
Missing the diagnosis of atrial septal defect (ASD) increases morbidities to patient and may require second surgery or intervention. Chronic constrictive pericarditis produces thickening and calcification of pericardium. The detection of any intracardiac lesion may be difficult by echocardiography due to the masking or shadowing effect of calcified pericardium. We report a case of 30-year-old male presented with congestive heart failure, dyspnea and abdominal swelling. Transthoracic echocardiography diagnosed constrictive pericarditis with no evidence of ASD. The contrast enhanced computed tomography (CECT) showed extensive diffuse pericardial calcification with a large ASD. In the operating room initial transesophageal echocardiography (TEE) examination was not able to detect any ASD. Agitated saline injected through the central venous line into right atrium showed bubbles in the left atrium under TEE monitoring. Subsequent movement of TEE probe in deeper position detected the ASD. It is recommended that all the views and methods of echocardiography examination may be practiced in difficult moments to avoid missing the presence of ASD.
\end{abstract}

Keywords: Transesophageal echocardiography, Chronic constrictive pericarditis, Atrial septal defect.

How to cite this article: Das S, Rajput RS. Coexistence of Chronic Constrictive Pericarditis can make the Echocardiographic Diagnosis of Atrial Septal Defect Challenging. J Perioper Echocardiogr 2013;1(2):66-68.

\section{Source of support: Nil}

Conflict of interest: None declared

\section{INTRODUCTION}

Chronic constrictive pericarditis (CCP) is the chronic inflammation between the two layers of pericardium, leading to a thick and calcified covering surrounding the heart. ${ }^{1}$ The thick pericardium impairs diastolic filling of the ventricles and reduced ventricular function. ${ }^{1}$ The diagnosis of $\mathrm{CCP}$ by all modalities of investigations creates difficulty in some occasion. ${ }^{2}$ The combined presence of CCP and atrial septal defect (ASD) is very rare. The detection of ASD in the presence of constrictive pericarditis might be difficult due to the thick calcified pericardium and equalization of pressure difference across interatrial septum. We report a case of difficulty in diagnosis of ASD by echocardiography in a patient with coexisting CCP.

\section{CASE HISTORY}

A 30-year-old male of $52 \mathrm{~kg}$ body weight presented with atrial fibrillation, congestive heart failure, dyspnea and abdominal swelling. Transthoracic echocardiography (TTE) examination revealed normal mitral, tricuspid, aortic and pulmonary valves. Tricuspid valve showed inflow variation of $35 \%$ with moderate tricuspid regurgitation during inspiration. Mitral valve showed inflow variation of more than $25 \%$ with prominent $\mathrm{E}$ wave with rapid early diastolic filling and short deceleration time of $95 \mathrm{msec}$ after inspiration. Patient had normal systolic function (left ventricular ejection fraction of 55-60\%), left ventricular hypertrophy and grade III diastolic dysfunction. Interventricular septum was bouncing toward left ventricle during inspiration, along with, left atrial and right ventricular enlargement. Right atrium was normal in collapsibility. Pericardium was $7 \mathrm{~mm}$ thick, calcified and effusion was present. Inspiratory diminished collapsibility of inferior vena cava $(13 \mathrm{~mm})$ was present; along with hepatic vein flow reversal during expiration. TTE did not show any evidence of intracardiac shunt defect.

The patient underwent contrast enhanced computed tomography (CECT) evaluation in direction of CCP. The CECT showed diffuse pericardial calcification predominantly anterior to right atria and right ventricle, biatrial dilatation with dilated IVC, right ventricle was dilated with a large ASD of size $1.6 \mathrm{~cm}^{2}$. There was normal systemic and pulmonary venous drainage.

The patient was planned for radical pericardiectomy keeping in mind that the ASD will be re-evaluated by TTE and transesophageal echocardiography (TEE) in the operation room. After induction of anesthesia, the TEE examination confirmed the presence of $\mathrm{CCP}$ with thick pericardium surrounding the heart with pericardial effusion (Fig. 1 and Video 1). The mid esophageal four-chamber view (Fig. 2 and Video 2) and the bicaval view (Fig. 3 and Video 3) was not able to detect any ASD. A thorough detail TEE examination was started. $10 \mathrm{ml}$ of agitated saline was injected through the central venous line into right atrium under TEE monitoring. The agitated bubbles were visible in the left atrium and gave the certainty for the presence of ASD. The TEE probe was introduced little deep with manual rotation of the probe to right and left sides. An ostium secondum type ASD of $1.5 \mathrm{~cm}$ in size was detected (Fig. 4 and Video 4). ASD closure was decided under cardiopulmonary bypass (CPB) after pericardiectomy.

In the operating room after radical pericardiectomy, ASD closure was performed with Dacron patch under CPB. 


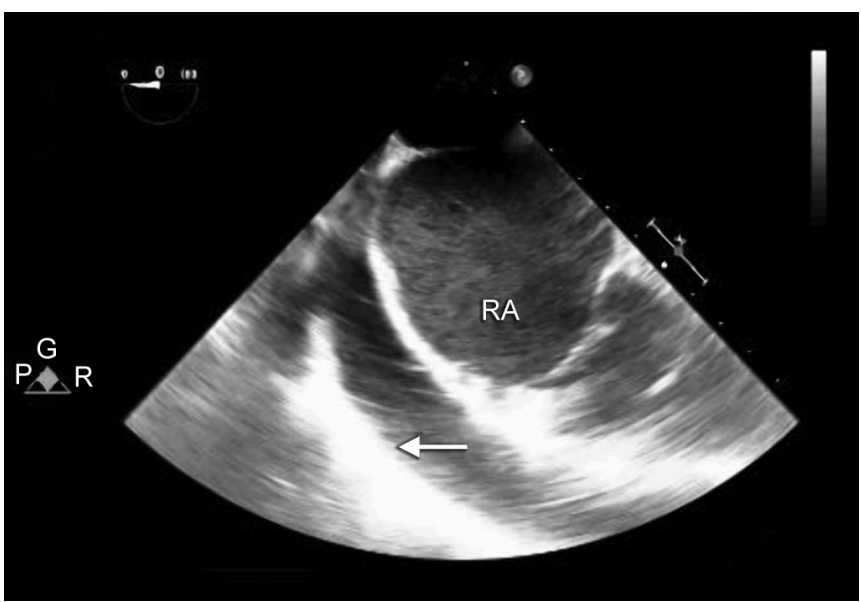

Fig. 1: TEE midesophageal position showing the thickened calcified (indicated with white arrow) pericardium (RA: Right atrium)
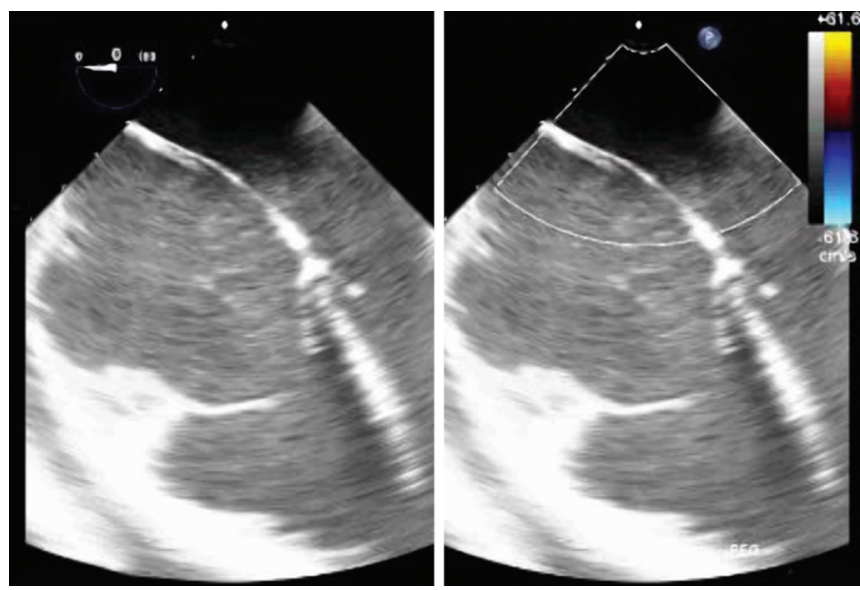

Fig. 2: TEE midesophageal four-chamber view color compare showing the interatrial septum with no defect
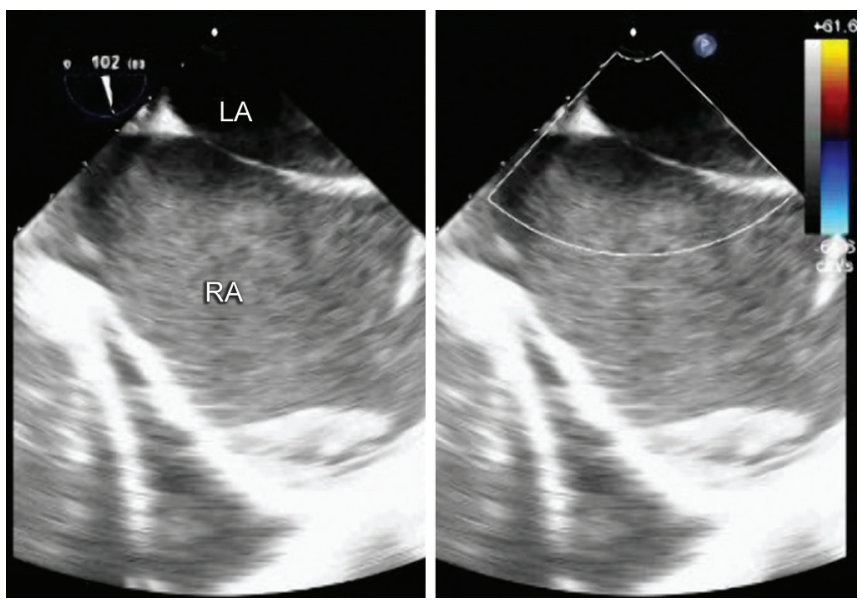

Fig. 3: TEE midesophageal bicaval view $\left(102^{\circ}\right)$ showing no defect across the interatrial septum (LA: Left atrium; RA: Right atrium)

The ASD was situated posteriorly in the interatrial septum near the coronary sinus. The complete closure of the ASD was confirmed by TEE. The postoperative course was smooth.

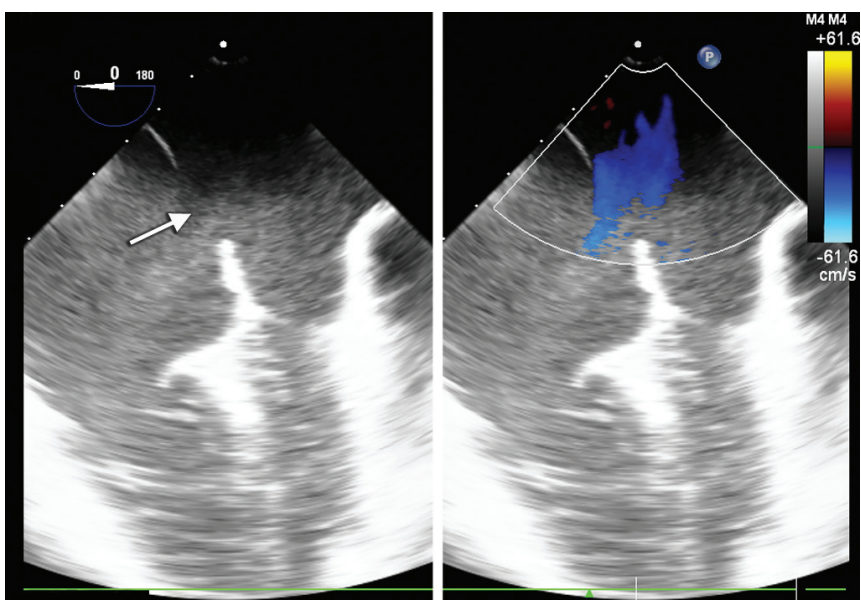

Fig. 4: TEE lower midesophageal four-chamber view color Doppler showing ASD (indicated with white arrow)

\section{DISCUSSION}

The combined association of CCP and ASD is quite uncommon. ${ }^{3}$ Tanoue et al reported a case of recurrent CCP and ASD in which the ASD was missed at the time of the first pericardiectomy. ${ }^{4}$ The diagnosis of CCP might be challenging by echocardiography when it exists alone; coexistence with an ASD can prove clinically misleading; sometimes, a complete diagnosis is made only at autopsy., 4 Clinicians should bear in mind that the coexistence of constrictive pericarditis and ASD may give rise to no color flow across the ASD because of equalization of pressure by the thick constricting pericardium. ${ }^{6}$ This only shows that there was no demonstrable ASD by color flow. Additional modalities are always needed such as bubble test with valsalva release to confirm the presence or absence of ASD.

Three-dimensional (3D) echocardiography might help to locate the ASD after cropping; however the presence of CCP will create difficulty to acquire an accurate image either in full volume or live 3D mode even with proper gain adjustment. ${ }^{7,8}$ The availability of $3 \mathrm{D}$ equipment and expertization of the examiner is necessary for this technique.

The probable cause for difficult diagnosis of ASD by preoperative TTE might be the interference from thick calcified pericardium and pericardial effusion in the anterior surface of heart. It would have been also difficult to visualize the posteriorly located ASD by TTE. The reasons for failure to detect the ASD in intraoperative initial TEE examination might be lack of flow across the interatrial septum and diffuse posterior pericardial calcification. The association of CCP with pericardial calcifications and atrial fibrillation might have interfered in locating the ASD by the superimposed shadow. The color Doppler might not able to pick up the flow across ASD due to no pressure difference between the two atria. The competency of the echocardiographer also 
plays a greater role in difficult situations. Hence a thorough echocardiographic evaluation with routine, contrast and agitated saline methods might be helpful in detecting difficult cases of ASD in patients with CCP. The complete diagnosis will minimize the number of surgical or interventional therapy to the patient.

\section{CONCLUSION}

The diagnosis of ASD in the presence of CCP might be difficult and missed in some occasion. The complete thorough evaluation by TEE with the use of contrast or agitated saline techniques will be helpful to detect the ASD.

\section{REFERENCES}

1. Spodick DH. Pericardial diseases. In: Braunwald E, Zippes DP, Libby P (Eds). Heart disease (6th ed). Philadelphia, London, Toronto, Montreal, Sydney, Tokyo: WB Saunders; 2001:182376.

2. Maisch B, Seferoviæ PM, Ristiæ AD, et al. Guidelines on the diagnosis and management of pericardial diseases executive summary: The task force on the diagnosis and management of pericardial diseases of the European society of cardiology. Eur Heart J 2004;25:587-610.

3. Uppu SC, Chandrasekaran S, Mallula KK. Constrictive pericarditis in a patient with sinus venosus atrial septal defect and anomalous right upper pulmonary venous return. Ann Pediatr Cardiol 2009 Jan;2(1):87-88.
4. Tanoue Y, Tomita Y, Kajiwara T, Tominaga R. Constrictive pericarditis with atrial septal defect. Ann Thorac Cardiovasc Surg 2006;12:373-75.

5. Kanda T, Naganuma F, Suzuki T, Murata K. Masked atrial septal defect in constrictive pericarditis. J Med 1993;24:325-32.

6. Edwin F, Tettey M, Sereboe L, Aniteye E, Tamatey M, EntsuaMensah K, et al. Atrial septal defect coexisting with constrictive pericarditis: A diagnostic conundrum. Internet $\mathrm{J}$ Thorac Cardiovasc Surg 2009;14(1).

7. Roberson DA, Cui W, Patel D, Tsang W, Sugeng L, Weinert $\mathrm{L}$, et al. Three-dimensional transesophageal echocardiography of atrial septal defect: A qualitative and quantitative anatomic study. J Am Soc Echocardiogr 2011;24(6):600-10.

8. Sharma VK, Radhakrishnan S, Shrivastava S. Three-dimensional transesophageal echocardiographic evaluation of atrial septal defects: A pictorial essay. Images Paediatr Cardiol 2011;13(3): $1-18$.

\section{ABOUT THE AUTHORS}

\section{Sambhunath Das}

Associate Professor, Department of Cardiac Anesthesia, All India Institute of Medical Sciences, New Delhi, India

Correspondence Address: 7th Floor, CN Centre, AIIMS, Ansari Nagar, New Delhi-110029, India, Fax: 91-11-26588663, 26588641 e-mail: sambhunathds833@gmail.com

\section{Randhir Singh Rajput}

Senior Resident, Department of Cardiac Anesthesia, All India Institute of Medical Sciences, New Delhi, India 